\title{
Neural Network Based Fault Detection and Diagnosis System for Three-Phase Inverter in Variable Speed Drive with Induction Motor
}

\author{
Furqan Asghar, ${ }^{1}$ Muhammad Talha, ${ }^{2}$ and Sung Ho Kim ${ }^{3}$ \\ ${ }^{1}$ Kunsan National University, Saemangeum Campus, Room No. 202/203, Osikdo-Dong, Gunsan-Si, \\ Jeollabuk-Do 573-540, Republic of Korea \\ ${ }^{2}$ School of Electronics and Information Engineering, Kunsan National University, Kunsan, Republic of Korea \\ ${ }^{3}$ Department of Control and Robotics Engineering, Kunsan National University, Kunsan, Republic of Korea \\ Correspondence should be addressed to Furqan Asghar; furqan.asghar@hotmail.com
}

Received 3 August 2016; Revised 5 October 2016; Accepted 12 October 2016

Academic Editor: Gang Li

Copyright (C) 2016 Furqan Asghar et al. This is an open access article distributed under the Creative Commons Attribution License, which permits unrestricted use, distribution, and reproduction in any medium, provided the original work is properly cited.

Recently, electrical drives generally associate inverter and induction machine. Therefore, inverter must be taken into consideration along with induction motor in order to provide a relevant and efficient diagnosis of these systems. Various faults in inverter may influence the system operation by unexpected maintenance, which increases the cost factor and reduces overall efficiency. In this paper, fault detection and diagnosis based on features extraction and neural network technique for three-phase inverter is presented. Basic purpose of this fault detection and diagnosis system is to detect single or multiple faults efficiently. Several features are extracted from the Clarke transformed output current and used in neural network as input for fault detection and diagnosis. Hence, some simulation study as well as hardware implementation and experimentation is carried out to verify the feasibility of the proposed scheme. Results show that the designed system not only detects faults easily, but also can effectively differentiate between multiple faults. These results prove the credibility and show the satisfactory performance of designed system. Results prove the supremacy of designed system over previous feature extraction fault systems as it can detect and diagnose faults in a single cycle as compared to previous multicycles detection with high accuracy.

\section{Introduction}

In recent years, induction motors are predominantly fed from pulse width modulation voltage source inverter (PWM-VSI) for variable speed operation in various industrial applications. Indeed, the most common drive in industry is that with a VSI and induction motor. Recently, industry has begun to demand high power ratings. Inverter drive systems have become a solution for high power applications as these systems are more reliable than those supplied directly online. There are several types of fault such as controller faults, current sensor faults, switching device faults, motor faults, and dc bus faults $[1,2]$. However, the use of inverters has some drawbacks as the introduction of power electronic converters came with an increased possibility of component failures mainly switching devices faults such as IGBT, MOSFET, and BJT.
These switching devices faults can be classified into open switch fault and short switch fault. A short switch fault not only generates an abnormal overcurrent in the power conversion system and generator but also causes some secondary problems like the demagnetization of synchronous generator. In this case, entire system should be shut down immediately for safety purpose, whereas an open switch fault does not require halting operation, but noise and vibrations can be induced in the system. Furthermore, the overcurrent flow in healthy switches can cause additional faults in these switches. Hence, open switch fault needs to be handled immediately. High costs due to standstill and repair, as well as general need to improve reliability, have led to research in fault detection systems [3-5].

Regarding diagnostics of these open switch faults, some of the previously researched approaches are mentioned here. 


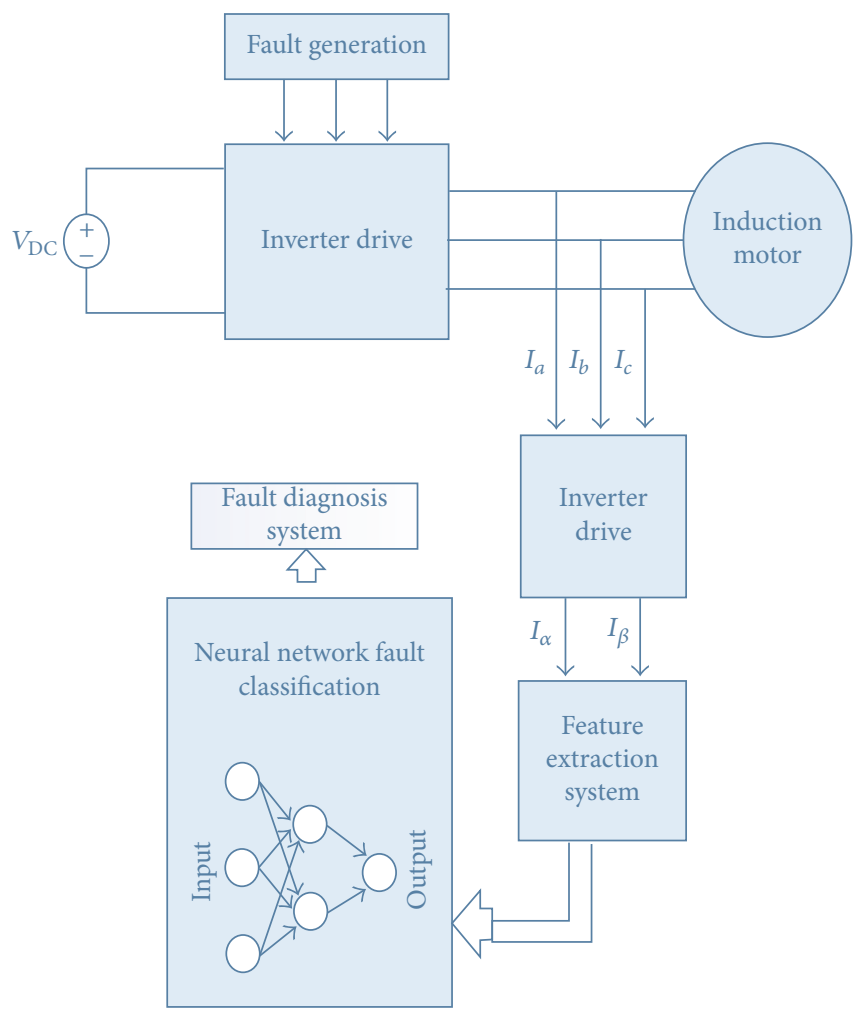

FIGURE 1: Fault detection and diagnosis system.

Peuget et al. suggested two methods for fault detection based on analysis of current vector trajectory and instantaneous frequency but technique using this frequency cannot detect faulty switches [6]. Khomfoi and Tolbert proposed a fault diagnostic technique to detect and identify fault location in multilevel inverter using neural network. However, this technique require computational effort [7]. Zidani et al. proposed a fuzzy based technique which detects faulty switches in PWM inverter for induction motor using Concordia pattern but this method was applied to single phase inverter [8]. Also Ko and Lee tried to propose fuzzy logic based fault diagnosis system but this technique is applicable only for single fault because fuzzy logic cannot differentiate between single and multiple faults angle as they overlap each other [9].

In this paper, neural network based fault detection and diagnosis method $[10,11]$ for three-phase inverter feeding an induction motor is designed to detect and localize failures in a set inverter-induction motor without the need of additional sensors or computational effort as shown in Figure 1. This technique can detect single or multiple switching device faults in three-phase inverter system by analyzing the stator current patterns and features extraction from that output current and then using these features in neural network method. Hardware implementation of simulation model is also carried out to confirm the feasibility of proposed scheme. Results proved that the designed fault detection and diagnosis system is more robust, accurate, systematic, effectual, and dynamic in detecting both single and multiple faults. This proposed technique is much better in comparison to previous techniques [7-10] as it can detect even multiple faults with
$100 \%$ accuracy because of efficient feature extraction system as compared to $95 \%$ or lower accuracy of those techniques, and also it can detect single and multiple faults faster even in single current cycle. These simulated and hardware based system results prove the credibility and show the satisfactory performance of system.

\section{Structure of Fault Detection and Diagnosis System}

Performance diagnosis and status monitoring for variable speed AC drives are a need, more or less depending on its applications. Fault detection and diagnosis can avoid unplanned maintenance and standstill, to make it possible to run an emergency operation in case of faults. We discussed two fault situations in this paper:

(i) Single fault

(ii) Multiple faults

2.1. Feature Extraction System. Feature extraction system must be determined as a system that can provide neural network adequate significant details in pattern set so that highest accuracy in neural network performance can be achieved. Feature extraction system should be universal for different speed references by normalized functions. Also localization of each pattern class should be in limits defined by threshold. In previous research studies, different researchers also try to use feature extraction system for fault detection 
and diagnosis for three-phase inverters and induction motors.

Ko and Lee tried to use feature extractor for his fault diagnosis system for inverter in wind turbine system [9]. He considered current angle and diameter as features with fuzzy logic technique for single fault detection only. This research work is not applicable for multiple faults as fuzzy logic will be unable to differentiate between single and multiple fault angles as they are overlapping each other. Therefore, further improvement is required to make this technique applicable for multiple faults detection.

Likewise, Zidani et al. [8] use the same angle and diameter difference in their research work along with fuzzy logic for fault detection. This approach is also applicable for only single fault detection.

Kadri et al. [10] also tried to use feature extraction for fault detection and diagnosis. But in their case, feature extractor is extracting only one feature as shown in

$$
S_{\alpha, \beta}=\frac{\sum_{i=1}^{N} I_{s_{\alpha, \beta}}(i)}{\operatorname{length}\left(I_{s_{\alpha, \beta}}\right) * \max \left(I_{s_{\alpha, \beta}}\right)} .
$$

This technique is acceptable only for constant speed environment; also they themselves mentioned that feature extraction system needs to be improved for better classification performance. System accuracy is not much high as single feature value can create false detections in case of multiple faults, that is, two or three faults at a same time.

In our proposed system, we used four different features along with neural network system for fault detection and diagnosis system which makes our system more accurate and efficient and separates it from previous techniques. High number of features play a vital role in differentiating single and multiple faults.

We used the below mentioned mathematical equations to calculate our four features for both Simulink and hardware environment separately. To get the features in faulty conditions, we generate faults in inverter system manually for every possible scenario. This process is repeated several times to consider every possible change in features values because of noise and other uncertainties in real time environment. Data range of each feature in every fault condition is determined based on repetitive process results in next step to utilize best possible data for neural network training. Then neural network is trained by this data for further process.

As shown in flow chart in Figure 1, designing ANN based on fault detection and diagnosis system consists of four major steps.

Initially the measured output current data is transformed from three-phase to two-phase using Clarke transformation. This transformation is performed to evaluate the stator current pattern evolution when open circuit power switches fault occur in the inverter as shown in Figure 2. Also, in Figure 3, fault diagnosis space for each switch fault is shown. In healthy and normal conditions, stator current pattern in $\alpha$ $\beta$ reference frame is a circle whereas, in case of fault, current pattern is biased towards the direction of faulty switch.

In feature extraction system, the significant assignment which is an analysis of the current is performed for detecting

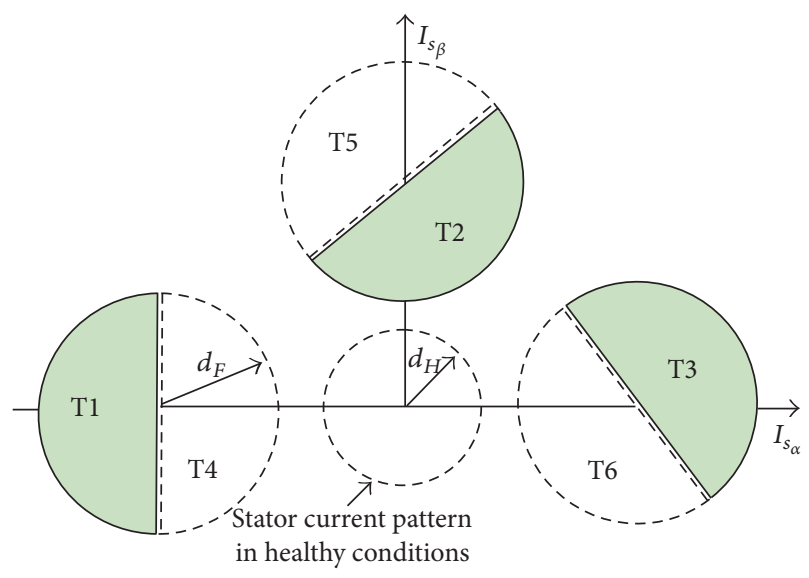

FIGURE 2: Current patterns in healthy and faulty modes.

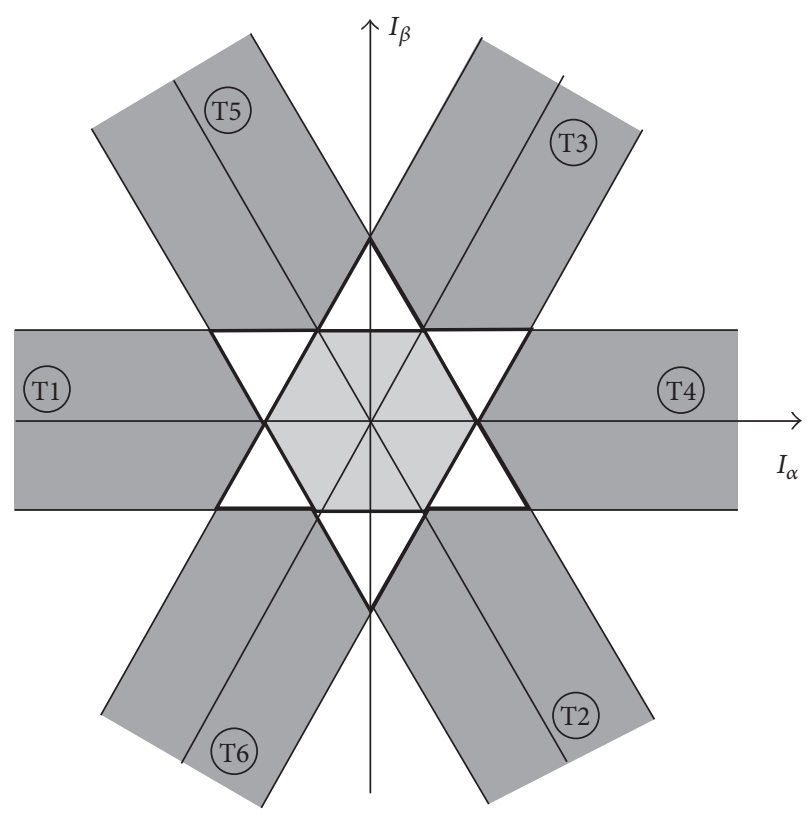

Figure 3: Fault detection and diagnosis space.

faulty switches. As mentioned before, transformation to stationary frame is done and various features from current pattern such as mean, surface, and angle are extracted.

During the fault detection step, faulty switches can be detected and identified using attributes obtained from the previous step. Output of this step will be 1 or 0 in which 0 represents off and 1 represent on to the respective state of switch.

Current means across both axes $(\alpha$ and $\beta$ ) can be calculated using the following equations:

$$
\begin{aligned}
& I_{\alpha}=\sum_{i=1}^{N} \frac{I_{\alpha}(i)}{\text { length }\left(I_{\alpha}\right)}, \\
& I_{\beta}=\sum_{i=1}^{N} \frac{I_{\beta}(i)}{\text { length }\left(I_{\beta}\right)},
\end{aligned}
$$



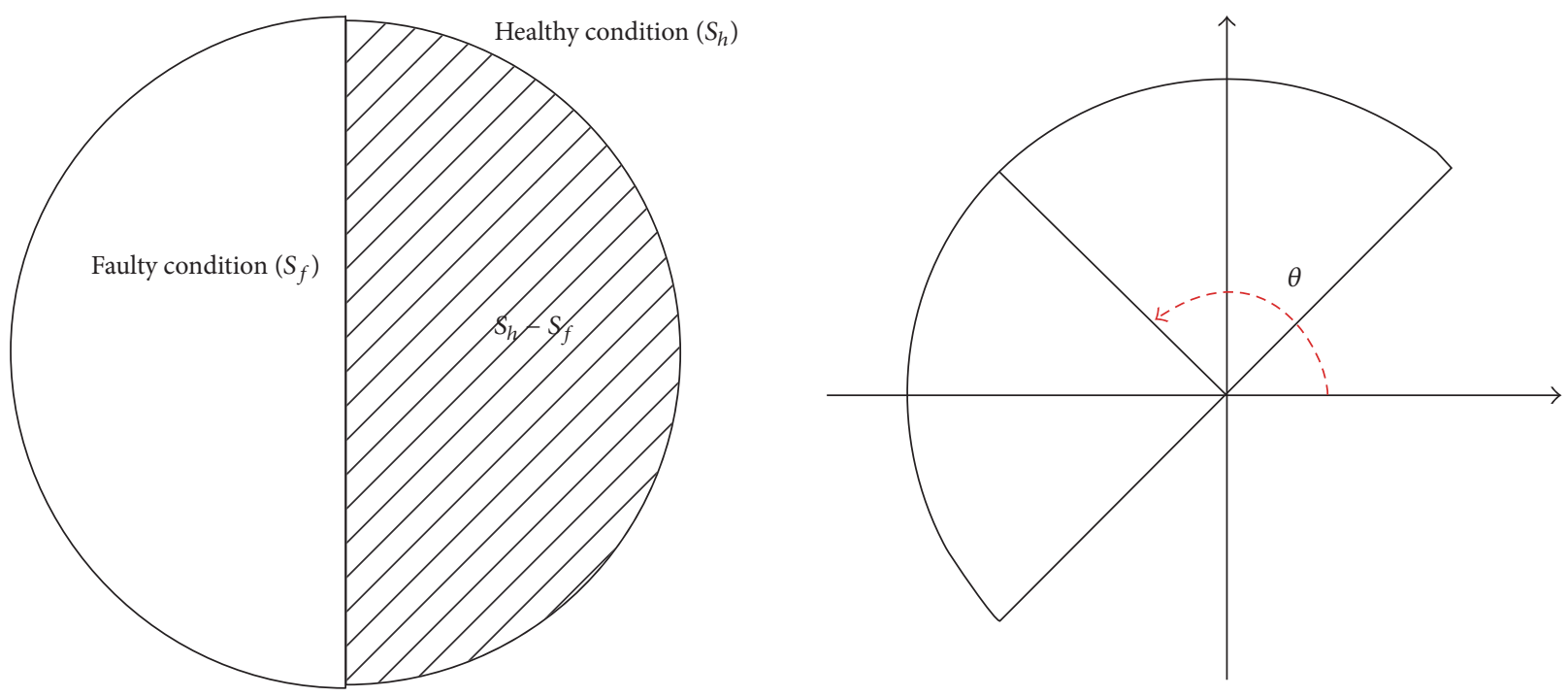

Figure 4: Definition of $E_{s}$ and $I_{\theta}$.

where $N$ defines the number of samples. Third feature angle to the current pattern $\left(I_{\theta}\right)$ can be calculated as

$$
I_{\theta}=\tan ^{-1}\left(\frac{I_{\beta}}{I_{\alpha}}\right),
$$

where $I_{\beta}$ is center of the $\beta$-frame and $I_{\alpha}$ is the center of $\alpha=$ frame. $I_{\theta}$ can be calculated by an angle between the origin and center of mass as shown in Figure 4.

Fourth feature surface difference of the current patterns between healthy and faulty condition shown in Figure 4 can be calculated as

$$
E_{s}=S_{h}-S_{f},
$$

where $S_{h}$ is current vector surface in healthy mode and $S_{f}$ is current vector surface in faulty mode.

Extracted features data shows that this block output gives an appropriate decorrelation between different kinds of single and multiple faults.

2.2. Artificial Neural Network System. Artificial neural networks (ANNs) are a family of models inspired by biological neural networks which are used to estimate or approximate functions that can depend on a large number of inputs and are generally unknown as shown in Figure 5.

Architecture of the designed fault detection and diagnosis neural network is a feed forward network as the input data contain continuous features. Our neural network is based on one input layer with four neurons each for one of four extracted features $\left(I_{\alpha}\right.$ (mean), $I_{\beta}$ (mean), angle, and surface difference), one hidden layer with 15 neurons, and one output layer with 13 neurons referring to the number of faults we want to detect. Sigmoid activation function is used for hidden and output layers. Target output of system is binary (1 or 0$)$.

Initially, neural network training is required with normal and faulty data. Then this trained neural network is used for fault detection system. Target output for normal case will be as follows:

$$
\begin{aligned}
& \text { Target Output } \\
& \quad=\left[\begin{array}{lllllllllllll}
1 & 0 & 0 & 0 & 0 & 0 & 0 & 0 & 0 & 0 & 0 & 0 & 0
\end{array}\right],
\end{aligned}
$$

where 1 represents the normal condition and 0's represent that currently there is no fault. Output will be 1 in case of respective fault such as for fault T1; output will be like as shown in

$$
\begin{aligned}
& \text { Target Output } \\
& \quad=\left[\begin{array}{lllllllllllll}
0 & 1 & 0 & 0 & 0 & 0 & 0 & 0 & 0 & 0 & 0 & 0 & 0
\end{array}\right] .
\end{aligned}
$$

\section{Simulation Studies}

Neural network based fault detection and diagnosis system for three-phase inverter with induction motor is briefly described in Section 2. Now the Matlab/Simulink simulation study is examined in this section to confirm its reliability.

3.1. Fault Generation. In our system, we generate fault in three-phase inverter system externally to check the performance of our proposed system in faulty conditions. We generate single and multiple faults by opening the IGBTs of inverter so that system can receive the input signal without respective phases. In case of double faults, usually there is high possibility of fault in two gates used in same phase like T1\&T2, T5\&T6, and so forth. Complete phase missing can be noticed in case of faults like short circuit or line to line fault. But being on the safe side, we trained our neural network for every possible scenario in two gate switch faults. Some of the generated faults are $\mathrm{T} 1, \mathrm{~T} 2, \mathrm{~T} 3, \mathrm{~T} 1 \& \mathrm{~T} 2, \mathrm{~T} 2 \& \mathrm{~T} 3$, $\mathrm{T} 4 \& \mathrm{~T} 6$, and so on.

3.2. Feature Data for Training Neural Network. In the start, we need to train neural network to work in efficient way 


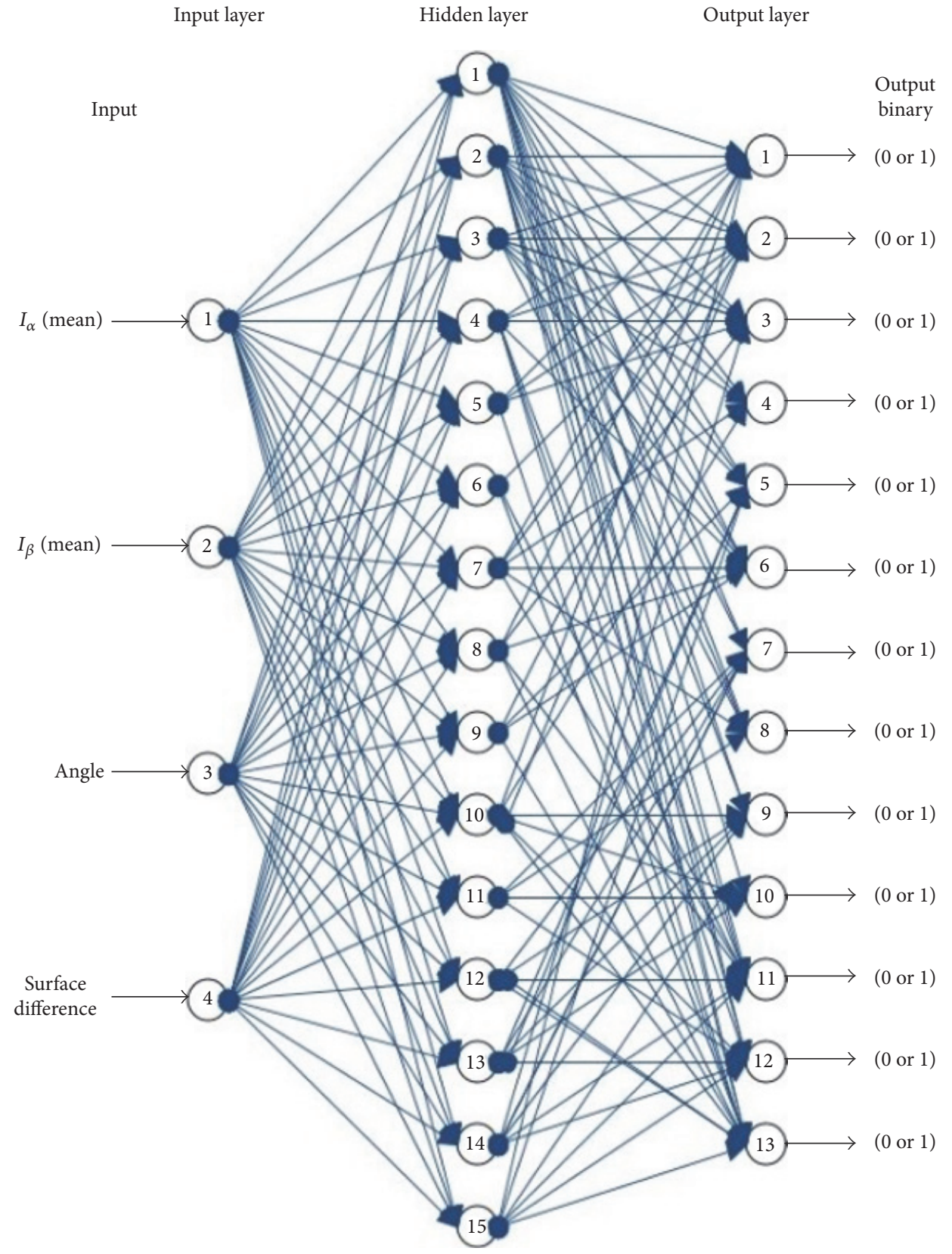

FIGURE 5: Basic architecture of artificial neural network.

according to desired environment. System requires normal and faulty feature data for training purposes as shown in Table 1.

After training neural network with the above-mentioned data, we can use this neural network system to detect faults in three-phase inverter feeding an induction motor. This system even works in case that extracted features in real time environment are not exactly the same as for training the network.

3.3. Simulation Results. Designed neural network based fault detection and diagnosis system for three-phase inverter in a variable speed drive is tested in case of single and multiple faults at a time. Simulink based system diagram can be seen in Figure 6.
In the simulation test sets, system shows satisfactory classification performance in both single and multiple faults cases.

Internal configuration of feature extraction block in normal mode can be seen in Figures 7 and 8 .

In Figure 7, we can see that artificial neural network block output is indicating that system is currently running in normal condition. Likewise, in Figure 8, $\alpha-\beta$ transformed current pattern graph shows circle which indicates system normal condition.

Designed system response can be seen in Figure 9 as it shows that system is working efficiently in both single and multiple switch fault modes.

Figures 10 and 11 show $\alpha-\beta$ transformed current pattern graph when system is operating in single fault whereas 


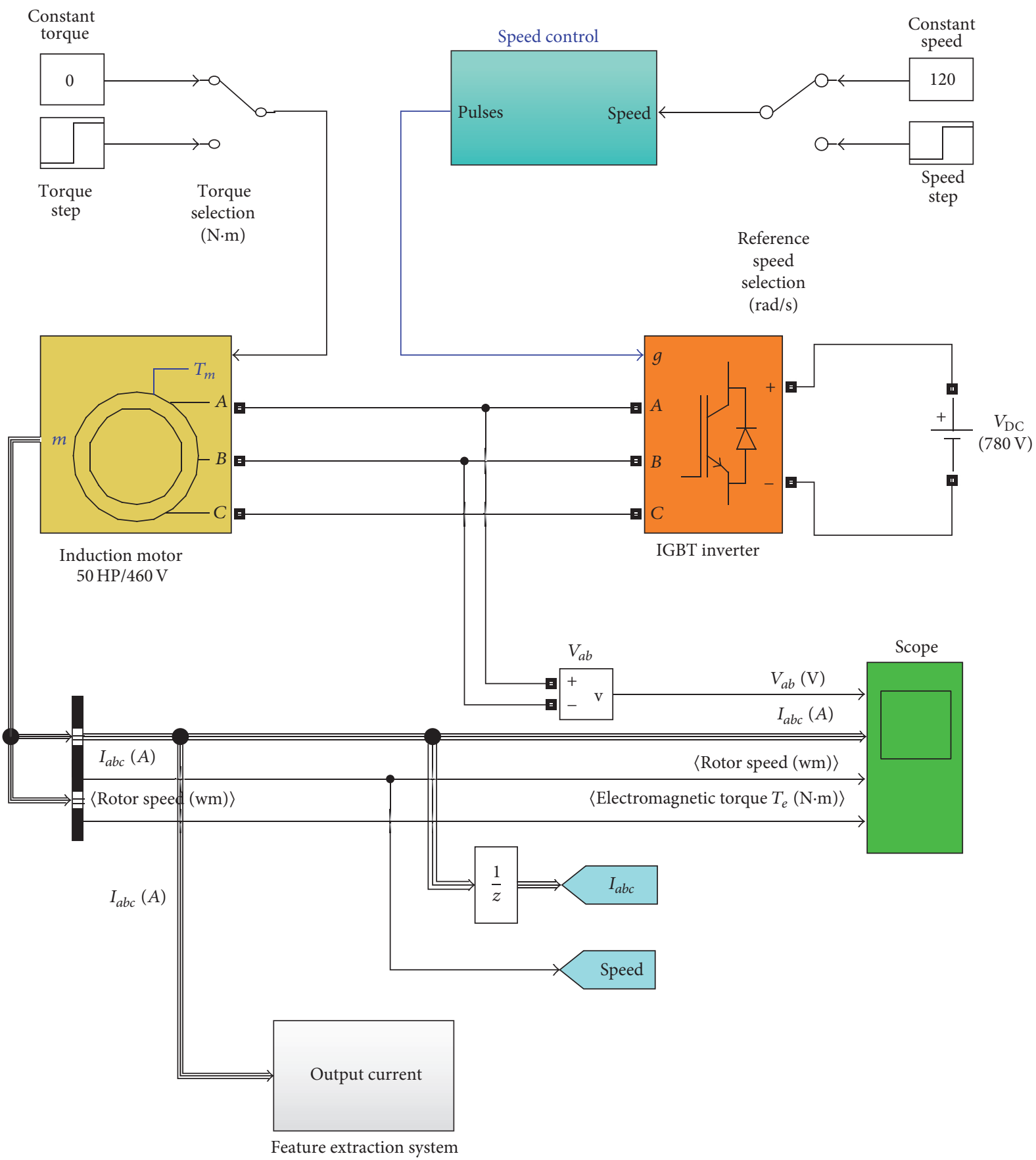

FIGURE 6: Neural network based fault detection and diagnosis system.

Figure 12 shows $\alpha$ - $\beta$ transformed current pattern graph during system operating in multiple faults, respectively.

3.4. Comparative Studies. As mentioned in Table 2 according to reference number of fault detection techniques, different researchers $[6-10,12]$ proposed fault detection and diagnosis techniques for inverters in the past. Some researchers designed their techniques for single phase inverters only.

Comparison of our technique with some of those methods shows that proposed fault detection is more robust and efficient than previously researched methods in both factors: accuracy and response time. As highest accuracy for above-mentioned previous fault detection methods is $95 \%$ at its best even most of these fault detection methods are for single fault, whereas, in our case, we are getting $100 \%$ accuracy even in multiple faults. Also proposed fault detection method can detect fault even in single current/voltage cycle whereas previous techniques need at least two cycles for fault detection.

\section{Hardware Implementation}

Proposed neural network based fault detection and diagnosis technique require hardware experimentation to prove their 


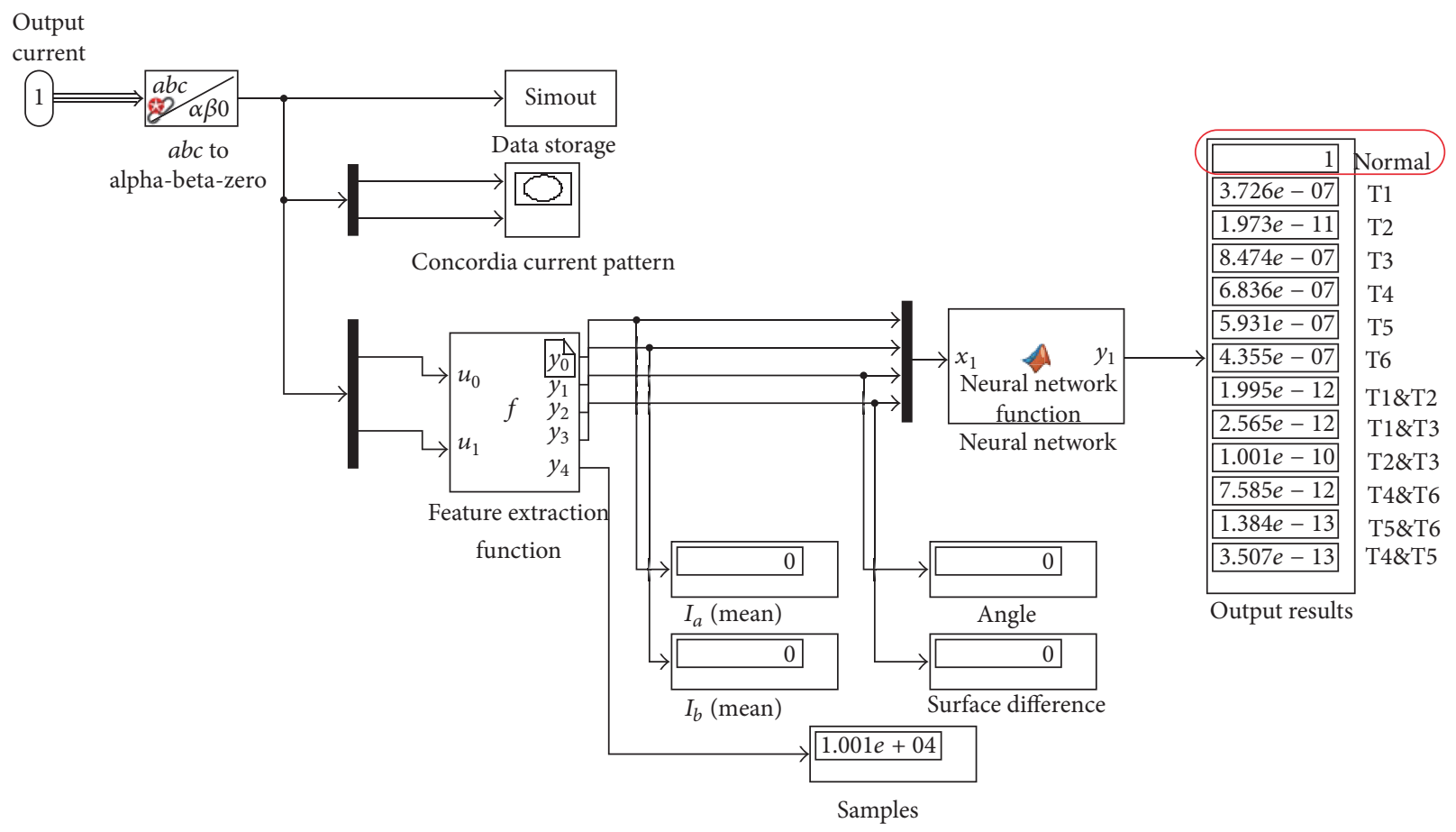

FIGURE 7: Internal configuration of feature extraction block with system normal mode.

TABLE 1: Feature data for training neural network.

\begin{tabular}{lcccc}
\hline System condition & \multicolumn{4}{c}{ Feature data for training } \\
States & $I_{\alpha}$ (mean) & $I_{\beta}$ (mean) & Angle & Surface \\
\hline Normal & 0.25 & 0 & 0 & 0 \\
T1 & -7.32 & -3.89 & 207 & 12.33 \\
T2 & 7.09 & -4.19 & 300 & 12.73 \\
T3 & 0.08 & 8.36 & 89 & 11.43 \\
T4 & 7.3 & 3.95 & 28 & 11.26 \\
T5 & -6.91 & 4.37 & 122 & 11.6 \\
T6 & -0.02 & -8.15 & 269 & 13.09 \\
T1\&T2 & -1.27 & -9.03 & 262 & 37.66 \\
T1\&T3 & 3 & 5.15 & 126 & 36.66 \\
T2\&T3 & 8.45 & 3.4 & 22 & 36.55 \\
T4\&T6 & 7.31 & -5.68 & 308 & 37.19 \\
T5\&T6 & -8.15 & -3.26 & 202 & 38.77 \\
T4\&T5 & 1.76 & 9.12 & 79 & 38.80 \\
\hline
\end{tabular}

accuracy and authenticity. Therefore, three-phase inverter is used to implement our proposed technique to compare the results with Simulink designed system output as shown in Figure 14. Three-phase inverter output voltages are used for feature extraction and further process.

SPWM inverter [13] is designed by using dsPIC30F4011 digital signal processing chip to generate switching pulses along with Lab-Volt (8134-20). .NET (C\#) based programing environment and monitoring system is used to design features extractor, artificial neural network (ANN), fault detection, and monitoring system. NI DAQ X-Series USB-6343

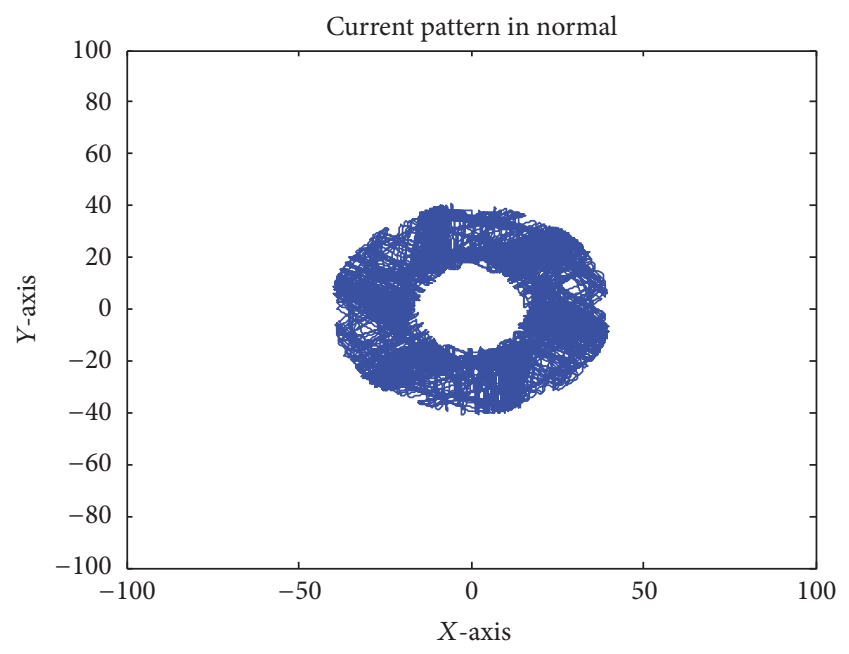

FIgURE 8: $\alpha$ - $\beta$ transformed current pattern graph for system in normal mode.

is used to acquire three-phase voltage signals from Lab-Volt output. System block diagram is shown in Figure 13.

4.1. SPWM Inverter Designing and Data Acquisition. dsPIC30F4011 is a high speed digital signal processing chip capable of generating high frequency PWM signals. Therefore, we use this chip to generate the gate pulses for three-phase inverter. Generated gate switching signals to three-phase inverter IGBTs are shown in Figure 15.

Lab-Volt is a training system with multipurpose modules that can be attached with various systems for experimentation 


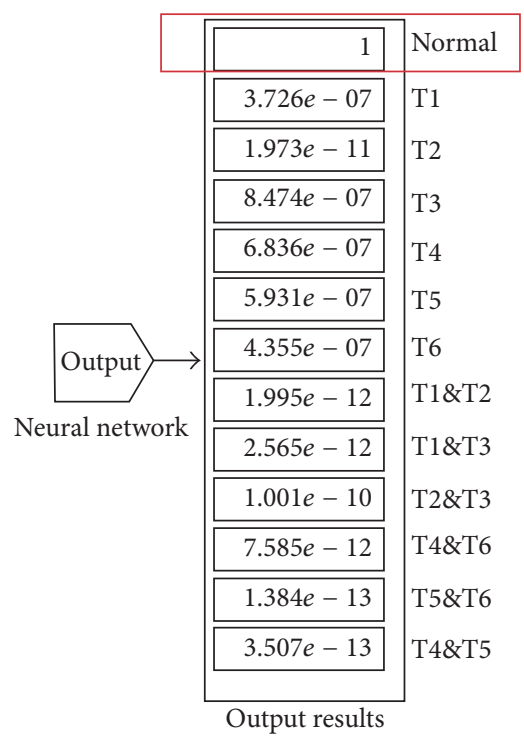

(a)

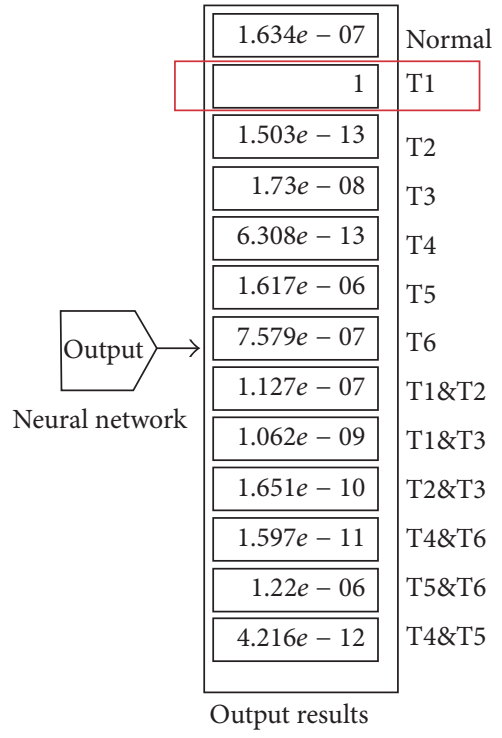

(b)

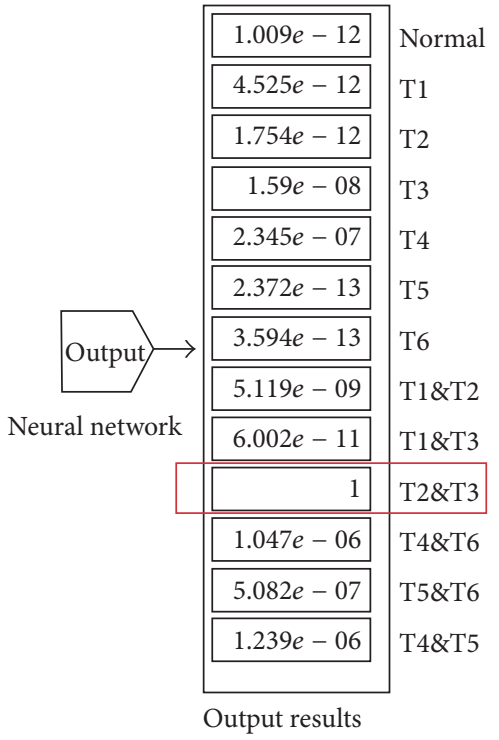

(c)

FIGURE 9: Simulation output results in various modes: (a) normal, (b) single fault, and (c) double fault.

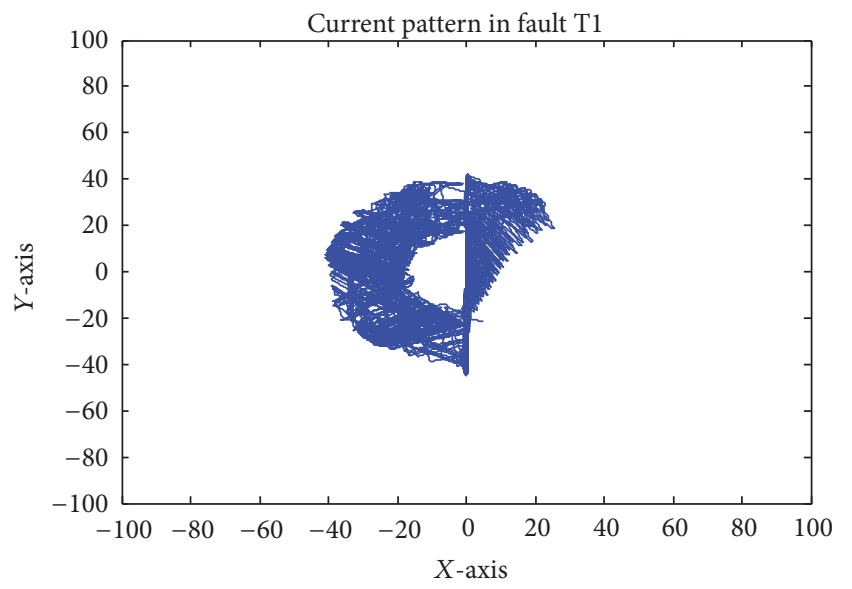

FIGURE 10: $\alpha-\beta$ transformed current pattern graph for system in single fault mode $\mathrm{T} 1$.

such as battery charging/discharging, data acquisition, Power MOSFETs and IGBTs, AC power interface, wind turbine emulator, and different types of loads such as resistive, capacitive, and inductive. Hence, Lab-Volt along with different modules is used to make three-phase inverter connected to RL load.

NI DAQ X-Series USB-6343 for USB, PCI express, and PXI express is one of the advanced data acquisition devices ever designed by National Instruments. Main features of NI DAQ X-Series USB are onboard timing, triggering, and optimization for use with multicore PCs. This device integrates high performance analog, digital, and counter/timer functionality onto a single device, making them well suited for a broad range of applications, from basic data logging to control and test automation. We are using this device to acquire three-phase output from Lab-Volt.

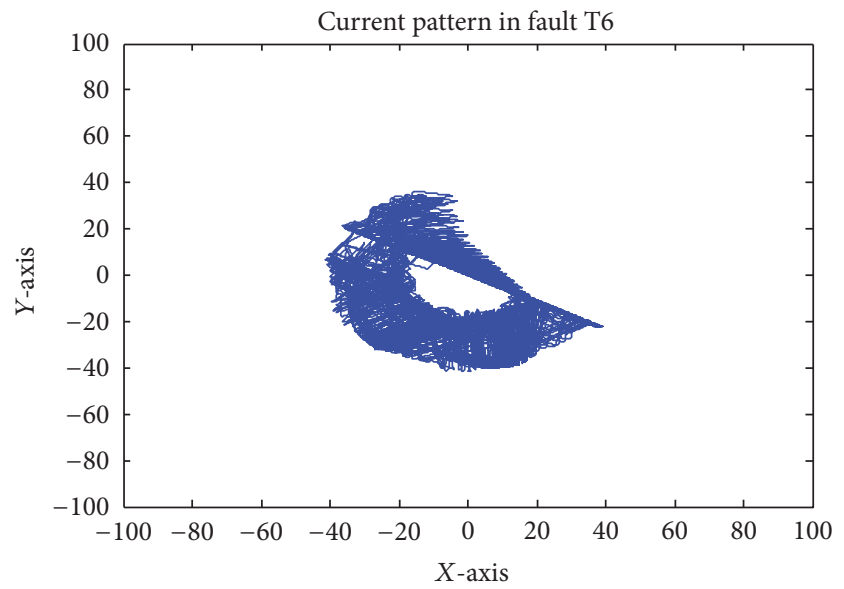

FIGURE 11: $\alpha-\beta$ transformed current pattern graph for system in single fault mode T6.

\subsection{Designing of .NET Based Fault Detection and Diagnosis} System. Microsoft visual studio is an integrated development environment used to develop computer programs as well as web applications and services. This IDE program supports different programming languages and allows the code editor to support almost every programming language. Built-in languages include $\mathrm{C}, \mathrm{C}++$, and C++/CLI, .NET, C\#, and F\#.

Three-phase voltage signal is being read in $\mathrm{C} \#$ program and then converted into two-phase using Clark Transformation $(\alpha, \beta$-axis). Various features are extracted from this twophase signal such as $I_{\alpha}$ (mean), $I_{\beta}$ (mean), pattern angle, and surface difference.

These four features are used to train artificial neural network in Matlab environment. Then trained artificial neural network is used in C\# program to detect single or multiple 


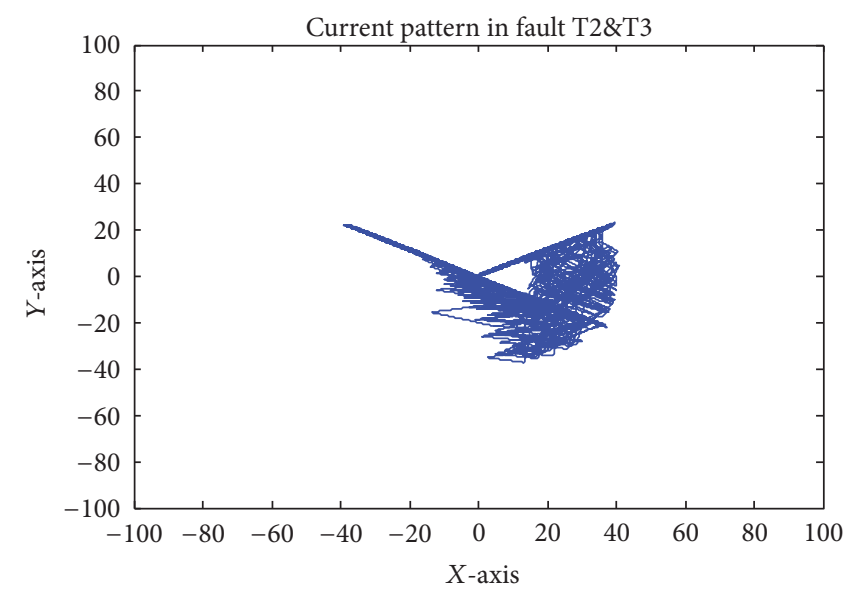

FIGURE 12: $\alpha-\beta$ transformed current pattern graph for system in multiple fault mode T2\&T3.

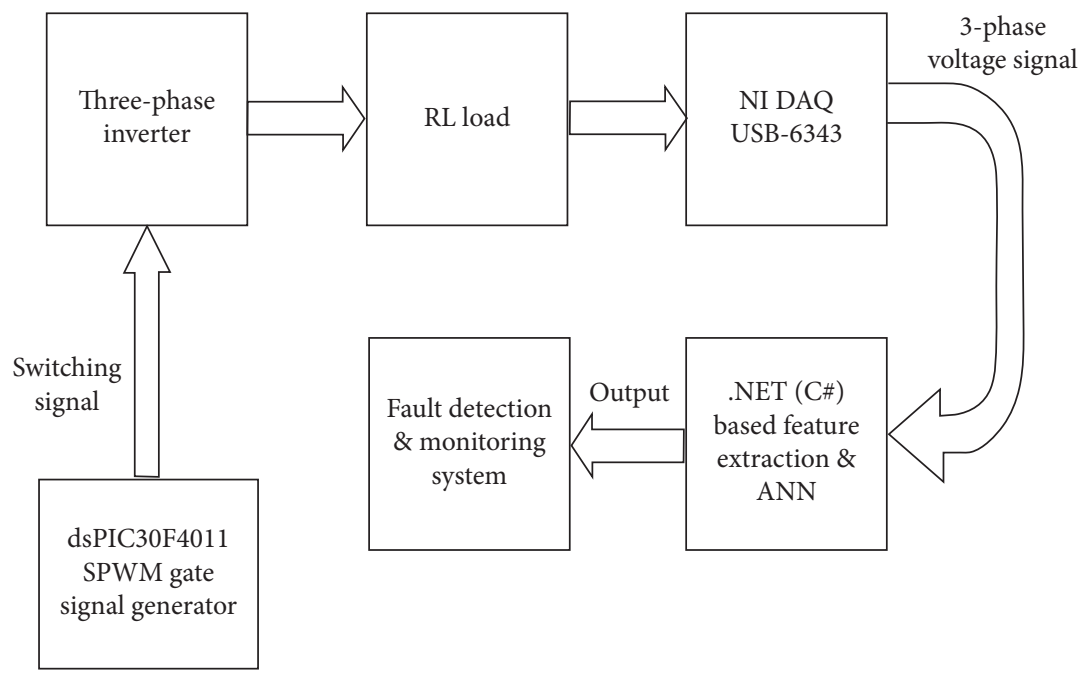

FIGURE 13: Hardware experimentation block diagram.

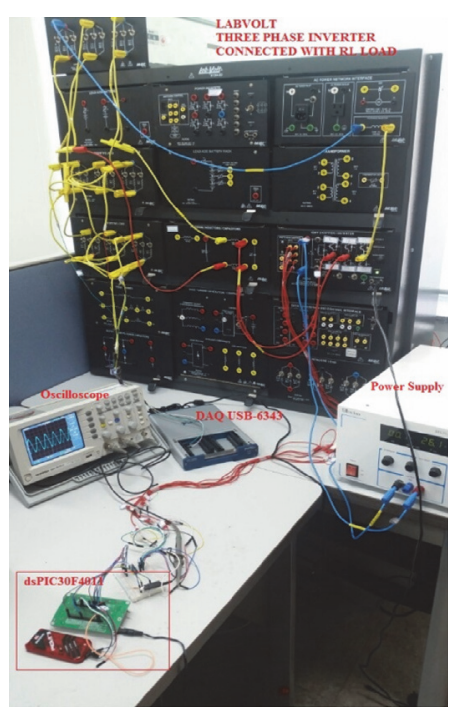

FIGURE 14: Experimental setup.

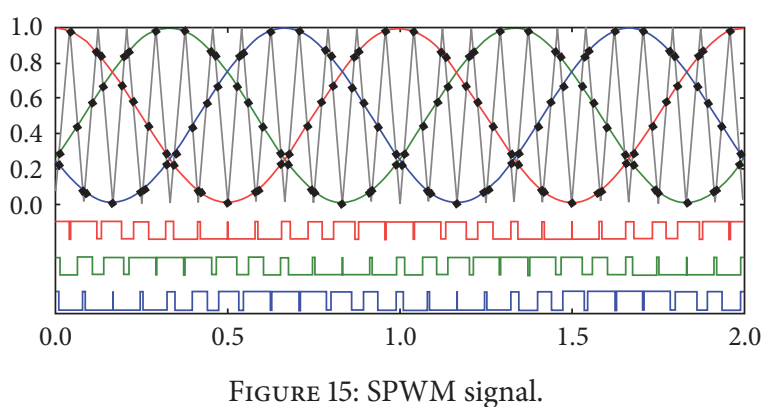

faults. Six DIP switches are connected to input of threephase inverter gate signals to add faults externally. Designed fault detection and diagnosis monitoring system are shown in Figure 16.

This figure shows that, in fault detection monitoring system, there are several options for better monitoring purposes such as loading three-phase output signal along with Clarke 
TABlE 2: Comparison table between different methods.

\begin{tabular}{lcccc}
\hline $\begin{array}{l}\text { Fault detection } \\
\text { Technique }\end{array}$ & Single & Accuracy & Response time & Inverter type \\
\hline Reference [6] & $100 \%$ & $<80 \%$ & Slow & 3 -phase \\
Reference [7] & $100 \%$ & $90 \%$ & Fast & 3 -phase \\
Reference [8] & $100 \%$ & Not applicable & Medium & 3 -phase \\
Reference [9] & $100 \%$ & Not applicable & Fast & 3 -phase \\
Reference [10] & $95 \%$ & $95 \%$ & Medium & 3 -phase \\
Reference [12] & $100 \%$ & $95 \%$ & Slow & 3 -phase \\
Proposed method & $100 \%$ & $100 \%$ & Fast & 3 -phase \\
\hline
\end{tabular}

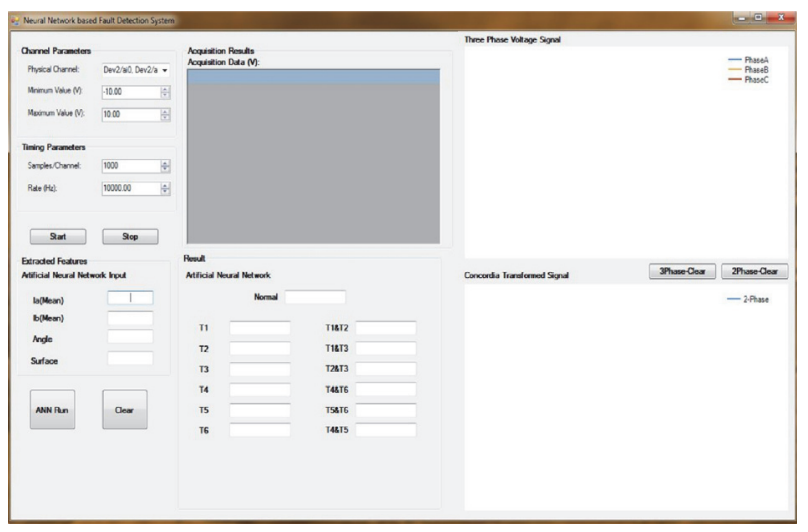

Figure 16: Artificial neural network based fault detection and diagnosis system.

transformed two-phase signal. Receive signal parameter and timing configuration setting are also available. Extracted features values can be monitored continuously.

4.3. Experimentation Results. Experimental setup as shown in Figure 14 is used to perform experiments with threephase inverter system running in various conditions such as normal, single fault, and multiple faults. Lab-Volt based threephase inverter can be seen connected with dsPIC30F4011 for gate switching signals, NI DAQ USB for output voltage acquisition, power supply, oscilloscope, and PC system for further processing. Inverter output voltages and frequency are constant, that is, $20 \mathrm{~V}_{\mathrm{p} \text {-p }}$ and $50 \mathrm{~Hz}$. Output voltage across load is acquired using NI DAQ in .NET based fault detection and diagnosis system. System response is shown in Figures 17,18 , and 19.

Figure 17 shows that three-phase inverter system is working in normal condition as we can see complete threephase voltage and circle in two-phase graph and normal condition is on in ANN output.

On the other hand, in Figure 18, system is indicating T5 fault as we generate T5 gate signal fault externally and also we can see the faulty condition in three-phase and two-phase voltage graph. Figure 19 shows that system is running with double fault at switches T4\&T6 simultaneously.

Experimentation results verified that proposed system is robust and accurate. Also it can detect and diagnose single and multiple faults efficiently.

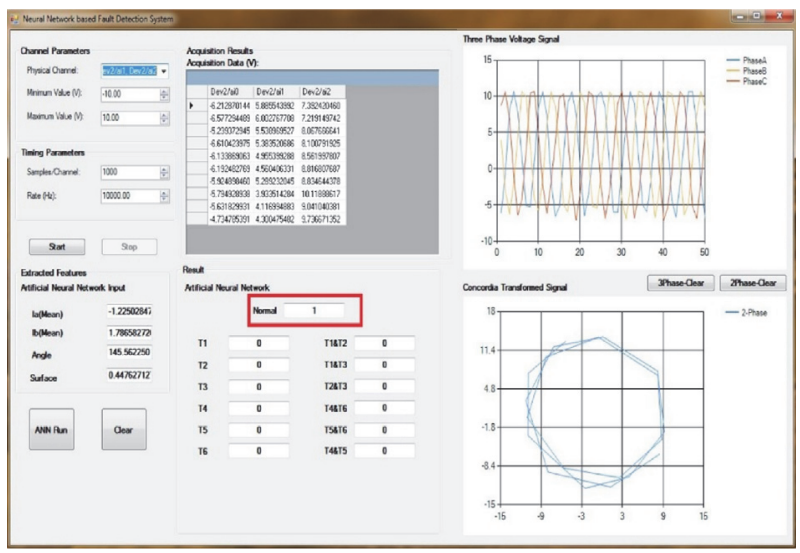

FIGURE 17: System operating in normal condition.

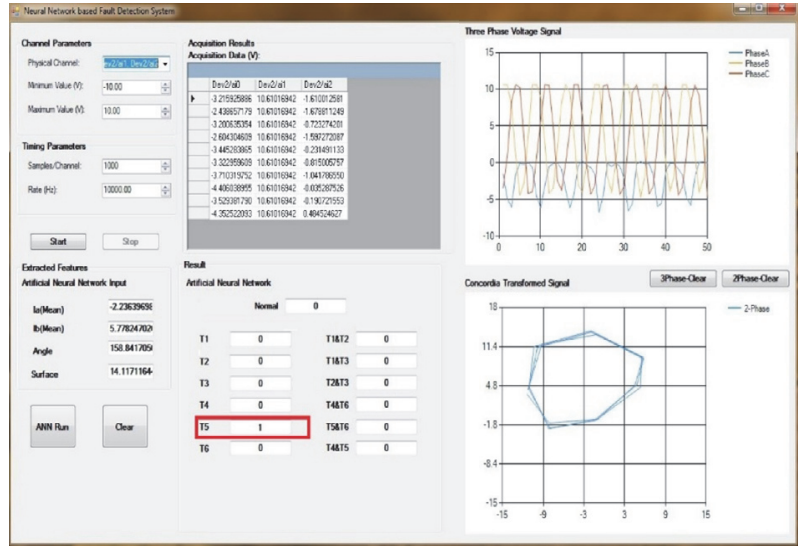

FIGURE 18: System operating in single fault (T5) condition.

Performance table for proposed neural network based fault detection and diagnosis system is also shown.

Simulation and hardware experimentation has been performed with every fault condition a number of times to verify the system accuracy. Data with respect to system condition is shown in Table 3.

\section{Conclusion}

In this research work, neural network based fault detection and diagnosis system for field oriented induction motor 
TABLE 3: Performance table for ANN based fault detection and diagnosis system.

\begin{tabular}{lllllllllllll}
\hline $\begin{array}{l}\text { Target } \\
\text { States }\end{array}$ & & \multicolumn{1}{c}{$\begin{array}{c}\text { Actual output } \\
\text { Neural network }\end{array}$} & \multicolumn{1}{c}{ Performance\% } \\
Experiment
\end{tabular}

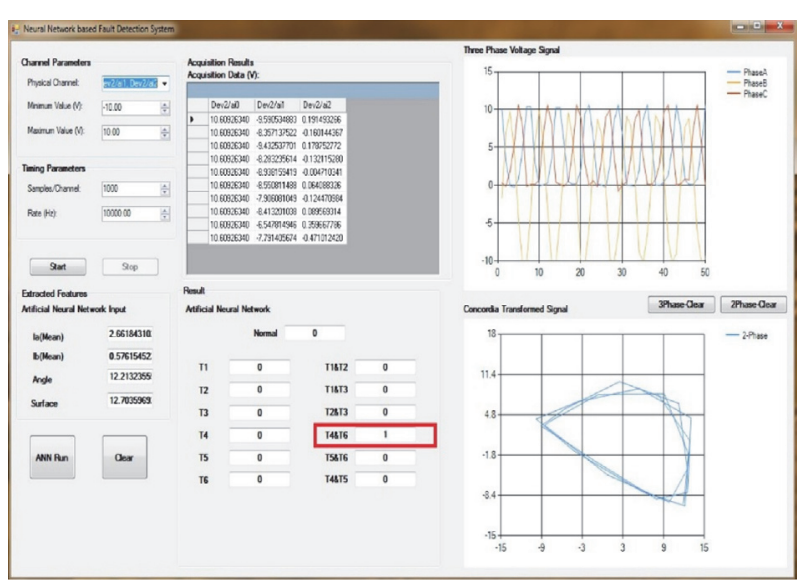

FIGURE 19: System operating in multiple faults (T4\&T6) condition.

drive have been designed and tested in both simulation and hardware environment. Considered fault types for system are switching device open faults. Feature extraction system based on current means, surface difference, and angle to current pattern is also discussed here. Extracted features play a vital role in fault detection and localization. A robust algorithm is needed for getting the correct angle because inaccurate value can lead to incorrect results. No additional sensors and complicated calculations are required for designed system. Additionally, faulty switch detection and identification can be performed even in every single current or voltage cycle with high accuracy which proves that system performance is much better than previous fault detection systems [6$10,12]$ as they take two or more cycles for fault detection. Simulated as well as hardware experimentation results as shown above prove the credibility and show the satisfactory performance of designed fault detection and diagnosis system for three-phase inverter feeding an induction motor. Multiple features extraction from three-phase current/voltage output signal plays a key role in proving proposed system supremacy over previous fault detection techniques. As shown in results, proposed system is fast, efficient, and 100\% accurate for single or multiple faults.

\section{Competing Interests}

The authors declare that there are no competing interests regarding the publication of this paper.

\section{References}

[1] D. Kastha and B. K. Bose, "Investigation of fault modes of voltage-fed inverter system for induction motor drive," IEEE Transactions on Industry Applications, vol. 30, no. 4, pp. 10281038, 1994.

[2] D. E. Kim and D. C. Lee, "Fault diagnosis of three phase PWM inverters using wavelet and SVM," Journal of Power Electronics, vol. 9, pp. 377-385, 2009.

[3] A. Muetze and A. Binder, "Practical rules for assessment of inverter-induced bearing currents in inverter-fed AC motors up to $500 \mathrm{~kW}$,' IEEE Transactions on Industrial Electronics, vol. 54, no. 3, pp. 1614-1622, 2007.

[4] M. R. Ubale, R. B. Dhmale, and S. D. Lokhande, "Open switch fault diagnosis in three phase inverter using diagnostic variable method," International Journal of Research in Engineering and Technology, vol. 2, no. 12, pp. 636-641, 2013.

[5] S. O. Ibrahim, K. N. Faris, and E. Abo Elzahab, "Implementation of fuzzy modeling system for faults detection and diagnosis in three phase induction motor drive system," Journal of Electrical Systems and Information Technology, vol. 2, no. 1, pp. 27-46, 2015.

[6] R. Peuget, S. Courtine, and J.-P. Rognon, "Fault detection and isolation on a pwm inverter by knowledge-based model," IEEE Transactions on Industry Applications, vol. 34, no. 6, pp. 13181326, 1998.

[7] S. Khomfoi and L. M. Tolbert, "Fault diagnostic system for a multilevel inverter using a neural network," IEEE Transactions on Power Electronics, vol. 22, no. 3, pp. 1062-1069, 2007.

[8] F. Zidani, D. Diallo, M. El Hachemi Benbouzid, and R. NaitSaid, "A fuzzy-based approach for the diagnosis of fault modes in a voltage-fed PWM inverter induction motor drive," IEEE 
Transactions on Industrial Electronics, vol. 55, no. 2, pp. 586-593, 2008.

[9] Y.-J. Ko and K.-B. Lee, "Fault diagnosis of a voltage-fed PWM inverter for a three-parallel power conversion system in a wind turbine," Journal of Power Electronics, vol. 10, no. 6, pp. 686-693, 2010.

[10] F. Kadri, S. Drid, F. Djeffal, and L. Chrifi-Alaoui, "Neural classification method in fault detection and diagnosis for voltage source inverter in variable speed drive with induction motor," in Proceedings of the 8th International Conference and Exhibition on Ecological Vehicles and Renewable Energies (EVER '13), pp. 15, Monte Carlo, Monaco, March 2013.

[11] V. Geometry and S. Selvaperumal, "Fault detection and classification with optimization techniques for three phase single inverter circuit," Journal of Power Electronics, vol. 16, pp. 1-14, 2016.

[12] S. Khomfoi and L. M. Tolbert, "Fault diagnosis and reconfiguration for multilevel inverter drive using AI-based techniques," IEEE Transactions on Industrial Electronics, vol. 54, no. 6, pp. 2954-2968, 2007.

[13] S. Phogat, "Analysis of single-phase SPWM inverter," International Journal of Science and Research, vol. 3, pp. 1793-1798, 2014. 


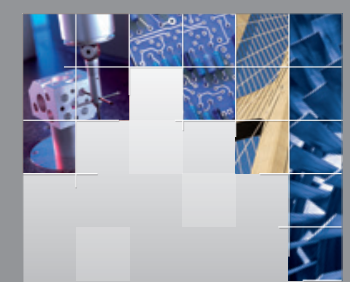

\section{Enfincering}
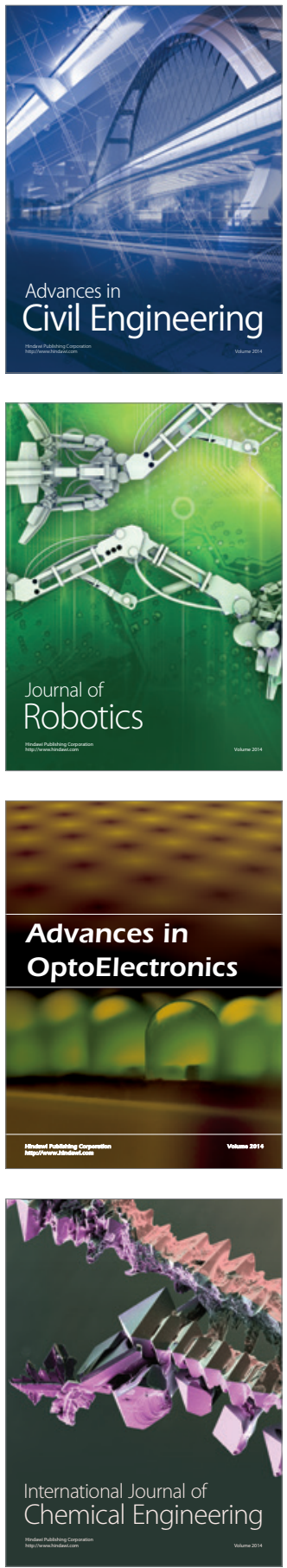

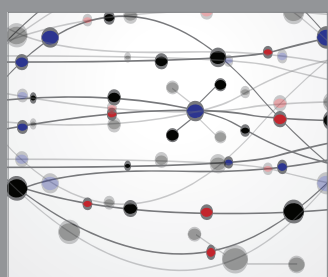

The Scientific World Journal

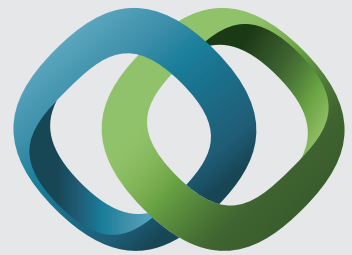

\section{Hindawi}

Submit your manuscripts at

http://www.hindawi.com
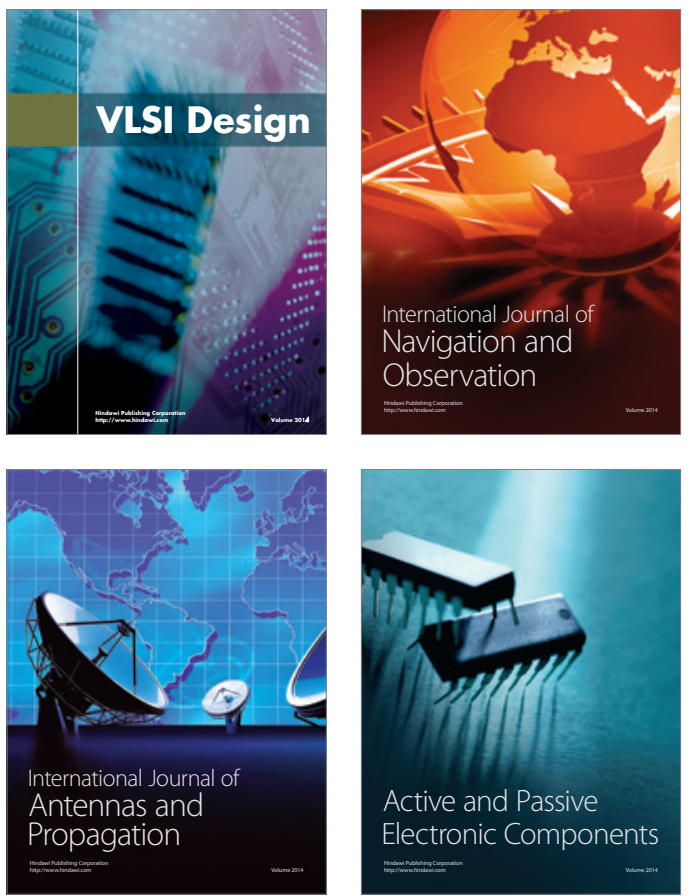
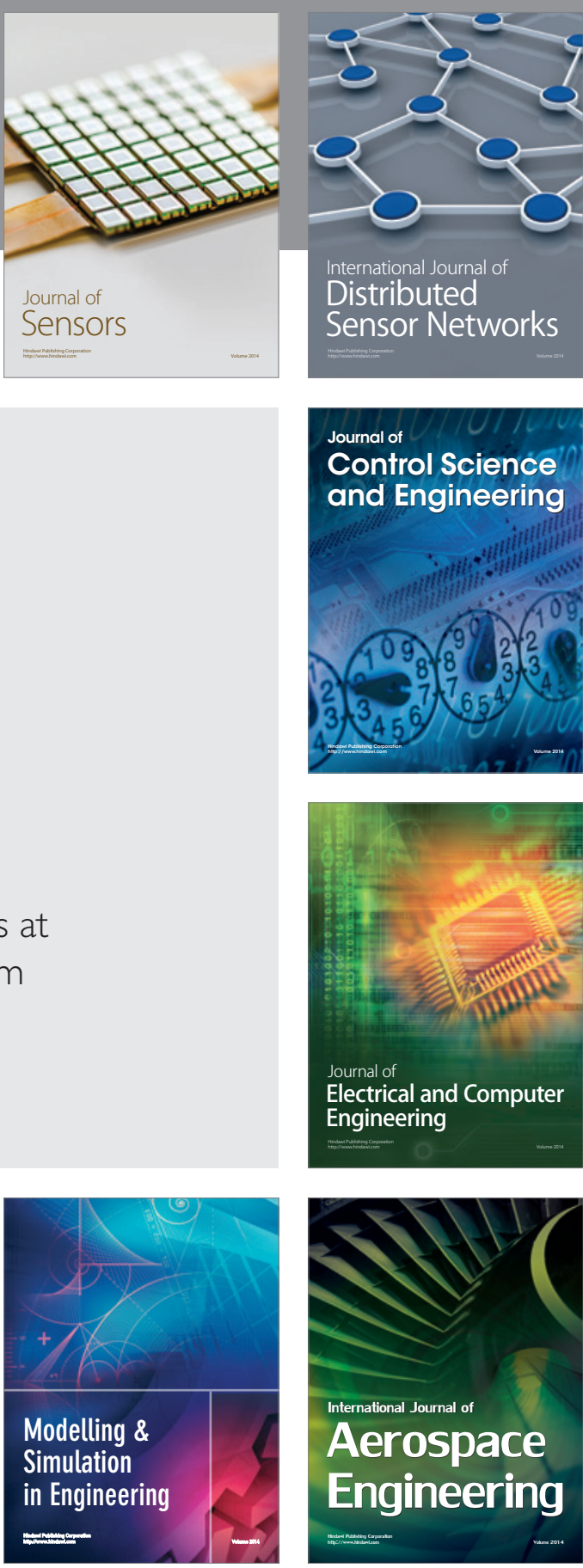

International Journal of

Distributed

Sensor Networks

Journal of

Control Science

and Engineering
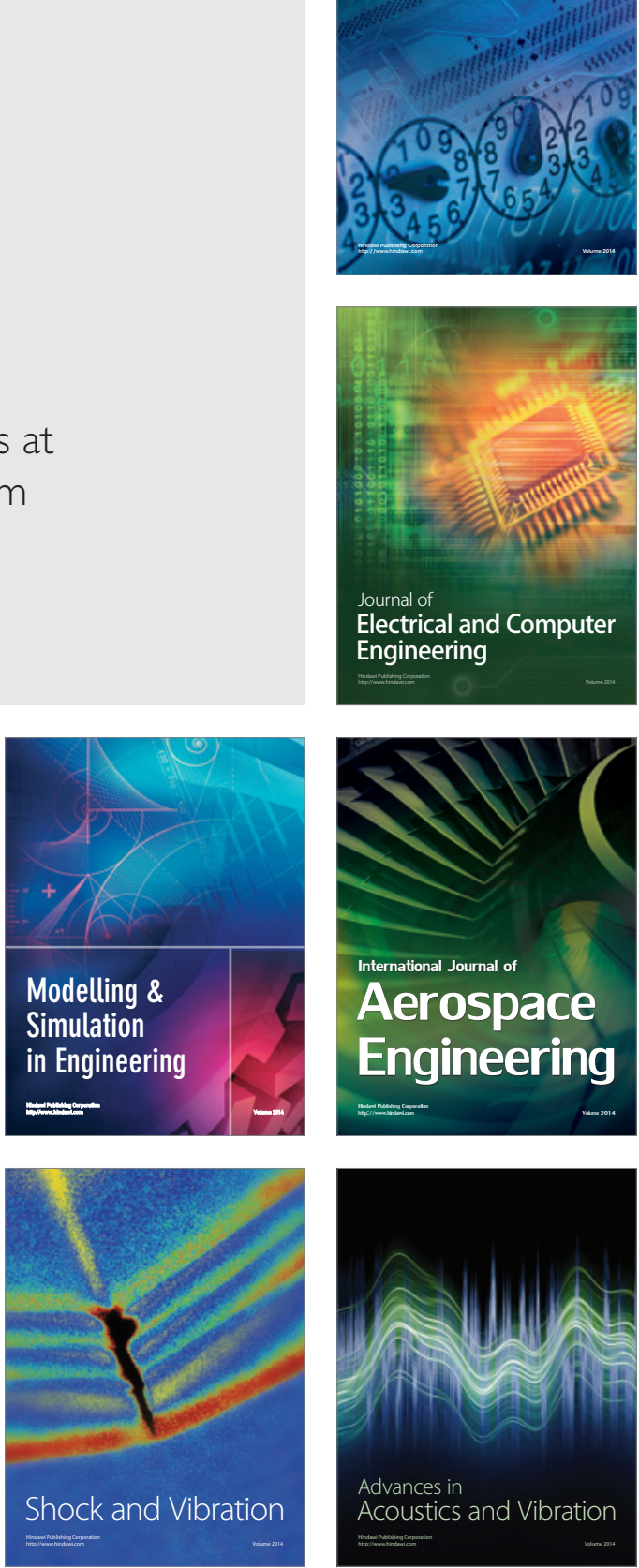